\title{
Puerarin inhibits non-small cell lung cancer cell growth via the induction of apoptosis
}

\author{
YEFANG HU ${ }^{1}, \mathrm{XILI}^{2}$, LING LIN ${ }^{1}$, SITING LIANG ${ }^{1}$ and JIAN YAN ${ }^{1}$ \\ ${ }^{1}$ Pharmacy Department, Guilin Traditional Chinese Medicine Hospital, Guilin, Guangxi 541002; \\ ${ }^{2}$ Key Laboratory for Chemistry and Molecular Engineering of Medicinal Resources, \\ Guangxi Normal University, Guilin, Guangxi 541004, P.R. China
}

Received May 29, 2017; Accepted December 12, 2017

DOI: $10.3892 /$ or.2018.6234

\begin{abstract}
Puerarin, an isoflavone isolated from Kudzu roots, has been demonstrated to have beneficial effect on cardiovascular and cerebral vascular diseases. Recently research has revealed that puerarin exerts an anticancer role in many different types of cancer. The aim of the present study was to investigate the antitumor effects of puerarin on non-small cell lung cancer (NSCLC). Treatment of puerarin significantly inhibited the growth of NSCLC cell lines as determined by CCK-8 kit in vitro. Flow cytometry results indicated that puerarin treatments promoted NSCLC cell apoptosis. This result was further confirmed by western blot analysis of expression levels of proteins involved in the mitochondrial-mediated apoptosis pathway. Moreover, puerarin slightly induced cell autophagy through the PI3K/Akt and MAPK/Erk1/2 signaling pathways. In addition, a tumor xenograft model was established using nude mice, and the inhibitory effects on tumor growth by puerarin treatment were also detected. Taken together, these findings demonstrated that puerarin has anticancer activities and puerarin is a potential therapeutic agent for lung cancer.
\end{abstract}

\section{Introduction}

Lung cancer is the most common cancer and the leading cause of cancer-related mortality in males worldwide with roughly 1.8 million new cases diagnosed in 2012 (13\% of all cancers) (1). Risk factors including exposure to environmental and occupational carcinogens have been associated with an increased incidence of lung cancer (2). It is well established that lung cancer is a clinically and pathologically heterogeneous disease and has been classified into two main histological types

Correspondence to: $\mathrm{Dr} \mathrm{Xi} \mathrm{Li}$, Key Laboratory for Chemistry and Molecular Engineering of Medicinal Resources, Guangxi Normal University, 2 Lingui Road, Guilin, Guangxi 541004, P.R. China E-mail: xi_li_china@hotmail.com

Key words: puerarin, non-small cell lung cancer, cell growth, apoptosis, autophagy non-small cell lung cancer (NSCLC) including squamous cell carcinoma, adenocarcinoma and large cell carcinoma and small cell lung cancer based on the origin of epithelial cell precursors (3). NSCLC accounts for $\sim 83 \%$ of all newly diagnosed lung cancers, and most patients $(70 \%)$ are diagnosed with advanced disease (4). Despite treatment advancements, many patients with recurrent disease fail to respond effectively to chemotherapy due to the development of resistance with treatment. Following treatment overall survival remains poor with 5-year survival estimates globally ranging from 10 to $20 \%$ (5-7). Therefore, it is critical to find new treatment options against this deadly disease.

Natural compounds, isolated and exploited from plants, have been reported to be involved in the modulation of several biological processes, thus showing a great potential to be translated into clinical use (8-10). Moreover, various members of these families of compounds have the ability to modulate signaling pathways as well as to regulate the expression of genes involved in cell cycle regulation, differentiation, and apoptosis (11). Besides being useful in prevention, some of these molecules could also be helpful for the treatment of cancer, especially in combination with other drugs $(12,13)$. Natural compounds often hold unusual structural features which cannot be easily mimicked (14). Therefore, such compounds may serve as an invaluable source of drug discovery for diverse diseases. However, despite the extensive attention focused on natural products, their exact regulatory mechanisms remain to be explored.

Puerarin (daidzein 8-C-glucoside, $\mathrm{C}_{21} \mathrm{H}_{20} \mathrm{C}_{9}$ ) is a major isoflavonoid compound, isolated from the herb Radix Puerariae $(15,16)$. Previous studies have shown that puerarin has beneficial effects on cardiovascular and cerebrovascular diseases, including coronary artery disease, heart failure, hypertension and myocardial infarction (17-19). In addition, recently studies have found that puerarin has an anticancer effect on tumor cells (20). Puerarin was found to inhibit HT29 cell growth by enhancing the anti-proliferative effects of other anti-neoplastic agents (21). Co-treatment of puerarin and 5-fluorouracil had a synergistic antitumor effect in gastric carcinoma (12). Moreover, puerarin inhibited cell growth and induced apoptosis in breast cancer and hepatocellular carcinoma cells $(22,23)$. Although puerarin inhibits the cell growth and induces apoptosis in many cancer types, there exists 
sparse research on the effectiveness of puerarin on NSCLC, and the underlying mechanism of its protective effects remains elusive. The aim of present study was to explore the inhibitory effect of puerarin on NSCLC cells, and elucidate the potential mechanisms of puerarin in lung cancer.

\section{Materials and methods}

Cell culture. NCI-H441 and NCI-H460 human lung adenocarcinoma cell lines were purchased from the American Type Culture Collection (ATCC; Manassas, VA, USA). Cells were authenticated on the basis of viability, recovery, growth and morphology. All cells were cultured in RPMI-1640 medium (Sigma-Aldrich, St. Louis, MO, USA), containing 10\% heatinactivated fetal bovine serum (Thermo Fisher Scientific, Inc., Waltham, MA, USA) at $37^{\circ} \mathrm{C}$ with $5 \% \mathrm{CO}_{2}$ in a tissue culture incubator. These cells are regularly tested to ensure that they are mycoplasma-free.

Chemicals and reagents. Puerarin was obtained from Sigma-Aldrich with a purity of $98 \%$, as assessed by reverse-phase high-performance liquid chromatography. Puerarin was stored at a $100 \mathrm{mM}$ concentration stock solution in dimethyl sulfoxide at $-20^{\circ} \mathrm{C}$ and diluted with serum-free culture medium for use in the experiments. PI3K/mTOR inhibitor rapamycin was purchased from Sigma-Aldrich, and BEZ235 (dactolisib) was obtained from Selleck Chemicals (Houston, TX, USA).

Cell growth inhibition studies. The inhibitory effect of puerarin on NCI-H441 or NCI-H460 cell growth in vitro was measured by Cell Counting Kit-8 (CCK-8; Sigma-Aldrich) according to the protocol of the manufacturer. Briefly, NCI-H441 and NCI-H460 cells were seeded in a 96-well plate at a density of 3,000 cells/well. Then the cells were treated with puerarin $(5,10$ and $20 \mu \mathrm{M})$ for different time-points. A volume of $1 / 10$ of the medium Cell Counting Kit solution was added to each well, and the plates were incubated for an additional $2 \mathrm{~h}$ (at $37^{\circ} \mathrm{C}$ with $5 \% \mathrm{CO}_{2}$ ). The optical density was then determined at a wavelength of $450 \mathrm{~nm}$ using a microplate reader (Bio-Rad Laboratories, Inc., Hercules, CA, USA). Each experiment was performed in triplicate and the results are presented as the inhibition rate (IR), which was calculated using the following formula: IR $(\%)=[(\mathrm{A}-\mathrm{B}) / \mathrm{A}] \times 100$, where $\mathrm{A}$ and $\mathrm{B}$ are the absorbance of the control and sample group following different time-points of incubation, respectively.

Annexin V/PI staining. Dead Cell Apoptosis kit with Annexin V-FITC and propidium iodide (PI) (Invitrogen, Carlsbad, CA, USA) was used according to the manufacturer's instructions to quantify the percentage of cells undergoing apoptosis. Briefly, NCI-H441 cells were exposured to puerarin $(5,10$ and $20 \mu \mathrm{M})$ for $72 \mathrm{~h}$. Next, the cells were washed twice with cold PBS and resuspended in binding buffer at a concentration of $1 \times 10^{6}$ cells $/ \mathrm{ml}$. Then, $5 \mu \mathrm{l}$ of Annexin V-FITC and $10 \mu \mathrm{l}$ PI were added, and the cells were incubated for $5 \mathrm{~min}$ at room temperature in dark. After incubation, $200 \mu 1$ of binding buffer was added and the cells were analyzed immediately by flow cytometry (BD Biosciences, Franklin Lakes, NJ, USA). The flow cytometric analysis was performed using CellQuest software (BD Biosciences). Annexin $\mathrm{V}^{+} / \mathrm{PI}^{-}$cells were identified as apoptotic cells, and Annexin $\mathrm{V}^{-} / \mathrm{PI}^{+}$cells were identified as necrotic cells. The entire procedure was repeated three times for each sample.

Western blot analysis. After treatment with puerarin for $72 \mathrm{~h}$, NCI-H441 cells were lysed, and total proteins were extracted. Protein concentration was determined using the BCA protein assay kit (Thermo Fisher Scientific, Inc.). After quantification, $30 \mu \mathrm{g}$ of protein was loaded for each group, and electrophoresis was performed on $10 \%$ polyacrylamide SDS gel. Following electrophoresis, the gel was blotted onto polyvinylidene fluoride membranes (Millipore, Billerica, MA, USA). After blocking in a $5 \%$ non-fat dry milk solution in washing buffer containing $10 \mathrm{mmol} / \mathrm{l}$ Tris (pH 7.5), $150 \mathrm{mM} \mathrm{NaCl}$, and $0.05 \%$ Tween-20, the membranes were incubated overnight at $4^{\circ} \mathrm{C}$ with different antibodies: anti-Bax (\#5023), anti-Bcl2 (\#3498), anti-cleaved caspase-3 (\#9664), anti-Atg5 (\#2630), anti-LC3 (\#12741), antiphospho-Akt (\#4060), anti-Akt (\#4691), anti-phospho-ERK (\#4370), anti-ERK (\#9102) and anti- $\beta$-actin (\#4970) (all dilutions, 1:1,000; all from Santa Cruz Biotechnology, Inc., Santa Cruz, CA, USA). After washing three times with washing buffer containing $0.05 \%$ Tween-20, the membranes were incubated for $2 \mathrm{~h}$ with horseradish peroxidase-coupled secondary antibodies at room temperature. Signals were detected using the ECL kit (Amersham Pharmacia Biotech; GE Healthcare, Chicago, IL, USA).

Monodansylcadaverine (MDC) staining. The autofluorescent agent monodansylcadaverine (MDC) (Sigma-Aldrich) was used as a specific autophagolysosome marker to analyze the autophagic process. After treatment with puerarin for $72 \mathrm{~h}, \mathrm{NCI}-\mathrm{H} 441$ cells were induced to autophagy by $100 \mathrm{nM}$ rapamycin, and then were incubated with $0.05 \mathrm{mM}$ MDC in PBS at $37^{\circ} \mathrm{C}$ for $10 \mathrm{~min}$. After incubation, the cells were washed three times with PBS and the quantitative analysis of MDC staining was assessed by flow cytometry (BD Biosciences).

Xenografts in nude mice. Six-week-old male BALB/c nu/nu mice were purchased from Beijing Vital River Laboratory Animal Technology Co. (Beijing, China) and maintained under specific pathogen-free conditions. All mice weighed 16-18 $\mathrm{g}$ and were bred in autoclaved, filter-top, microisolator cages, which were kept in an isolator unit with filtered air. The mice had access to water and food ad libitum. The mice were inoculated subcutaneously with $1 \times 10^{7} \mathrm{NCI}-\mathrm{H} 441$ cells per mouse and the tumor sizes were measured using micrometer calipers. Puerarin was solubilized in normal saline buffer. When tumors grew to a mean volume of $100 \mathrm{~mm}^{3}$, the mice were randomly divided into the following four groups $(n=6 /$ group): normal saline (vehicle), puerarin $(25 \mathrm{mg} / \mathrm{kg} / \mathrm{day})$, puerarin $(50 \mathrm{mg} / \mathrm{kg} / \mathrm{day})$, and puerarin $(100 \mathrm{mg} / \mathrm{kg} / \mathrm{day})$, which was injected by oral gavage daily for 5 weeks. The tumor volume (TV) was calculated using the following formula: TV $\left(\mathrm{mm}^{3}\right)=\mathrm{d}^{2} \mathrm{x}(\mathrm{D} / 2)$, where $\mathrm{d}$ and $\mathrm{D}$ are the shortest and longest diameters, respectively. The tumor sizes and tumor weights of the mice were measured weekly. The mice were sacrificed by using $\mathrm{CO}_{2}$ asphyxiation at the end of 5 weeks and the tumors were removed, weighed and measured for additional analyses. All animal procedures, which complied with the National 
A<smiles>O=c1c(-c2ccc(O)cc2)coc2c([C@@H]3O[C@H](CO)[C@@H](O)[C@H](O)[C@H]3O)c(O)ccc12</smiles>

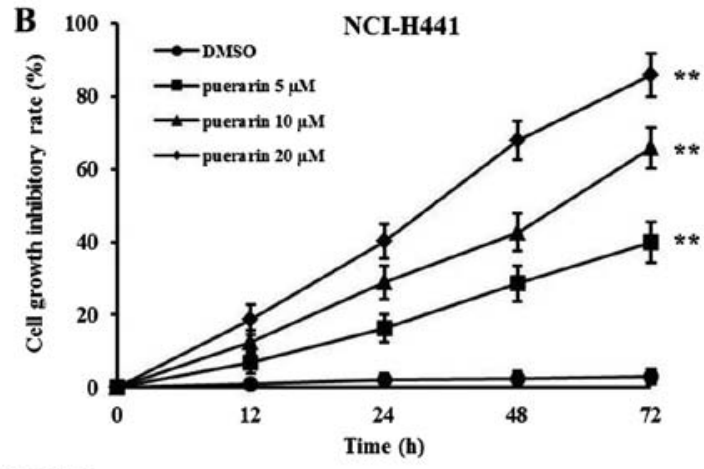

NCI-H441

C

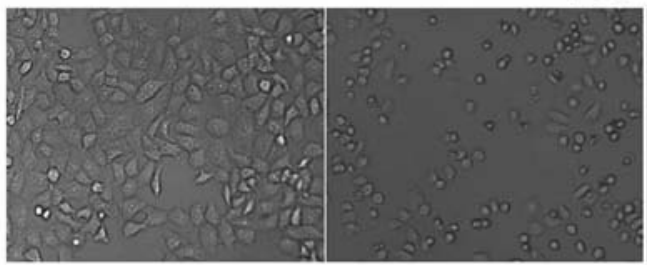

5

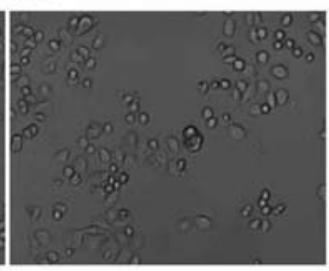

10

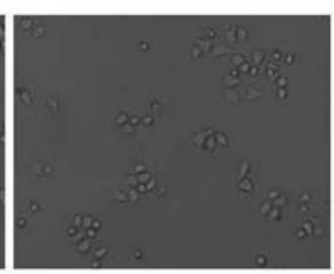

20

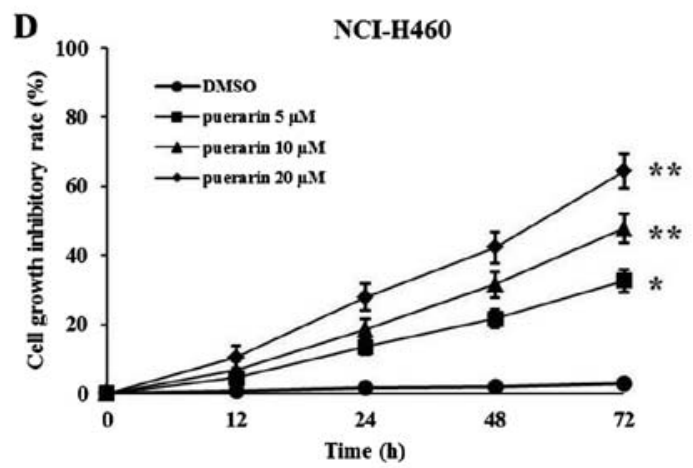

Figure 1. Effect of puerarin on lung carcinoma cell growth. (A) The chemical structure of puerarin. (B) The cell growth of NCI-H441 cells was inhibited by puerarin treatment in a dose-dependent manner $\left({ }^{* *} \mathrm{P}<0.01\right.$ compared with the DMSO control, $\mathrm{n}=3$ ). (C) Representative images of cell number and morphology for each group were captured under a microscope. (D) The cell growth of NCI-H460 cells was inhibited by puerarin treatment in a dose-dependent manner $\left({ }^{*} \mathrm{P}<0.05,{ }^{* *} \mathrm{P}<0.01\right.$ compared with the DMSO control, $\left.\mathrm{n}=3\right)$.

Institutes of Health Guide for the Care and Use of Laboratory Animals (24), were approved by the Committee on Animal Experimentation of Guangxi Normal University.

TUNEL assay. Sections of each tumor xenograft were fixed in $4 \%$ formaldehyde, dehydrated with an ethanol gradient, embedded in paraffin, dewaxed and rehydrated with a decreasing ethanol gradient (100, 95, 90, 80 and 70\%), according to standard instructions. An in situ apoptosis detection kit (Roche Molecular Systems Inc., Branchburg, NJ, USA) was used to detect apoptosis. All procedures were performed according to the manufacturer's instructions. The specimens were incubated with proteinase $\mathrm{K}[15 \mu \mathrm{g} / \mathrm{ml}$ in $10 \mathrm{mM}$ Tris/ $\mathrm{HCl}(\mathrm{pH} \mathrm{7.5)}]$ for $20 \mathrm{~min}$ at room temperature after being dewaxed and rehydrated. Next, the specimens were rinsed with $3 \% \mathrm{H}_{2} \mathrm{O}_{2}$, and incubated with equilibration buffer and terminal deoxynucleotidyl transferase (Beyotime Institute of Biotechnology). The specimens were then incubated with an anti-digoxigenin-peroxidase conjugate. Finally, the 3,3'-diaminobenzidine substrate was added to react with the peroxidase and the specimens were counterstained with hematoxylin, mounted and observed under a microscope.
Statistical analysis. All values are presented as the mean \pm standard deviation. Differences were determined by two-tailed Student's t-test and one-way ANOVA using the SPSS 20.0 statistical software (SPSS, Inc., Chicago, IL, USA). P-values of $<0.05$ were indicative of a statistically significant result.

\section{Results}

Puerarin inhibits lung carcinoma cell growth. The structure of puerarin is shown in Fig. 1A. To evaluate the effects of puerarin on cell growth, human lung adenocarcinoma NCI-H441 cells were treated with different concentrations of puerarin, and cell viability was determined by Cell Counting Kit-8 (CCK-8) at different times after treatment. As shown in Fig. 1B, puerarin inhibited the growth of NCI-H441 cells in a time- and dose-dependent manner (with increasing concentrations from 5 to $20 \mu \mathrm{M}$ ), and showed significant inhibition at concentrations of 10 and $20 \mu \mathrm{M}$ after puerarin treatment for 24,48 and $72 \mathrm{~h}$. Cell morphology and density were also observed under a microscope after a 72-h treatment at the concentration of $20 \mu \mathrm{M}$. Consistent with the cell growth results, there was a significant reduced cell density in the puerarin-treated cells 
A

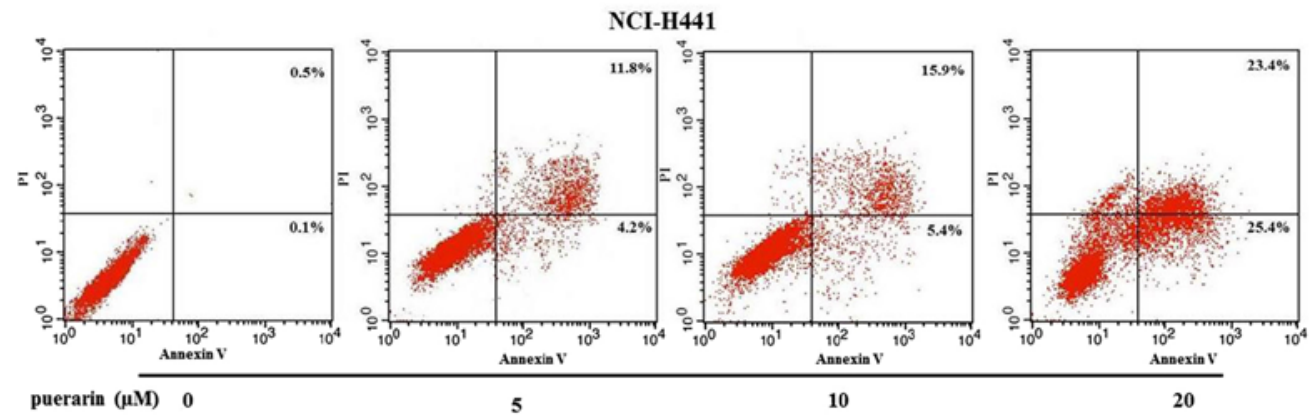

B

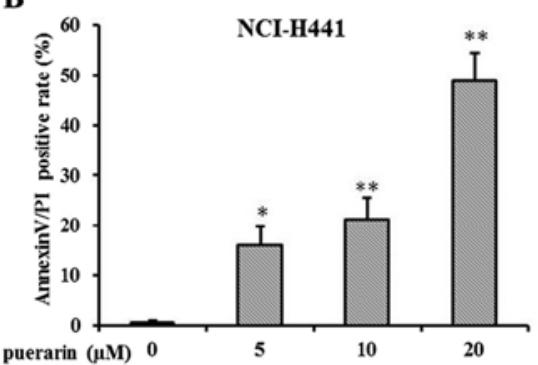

E

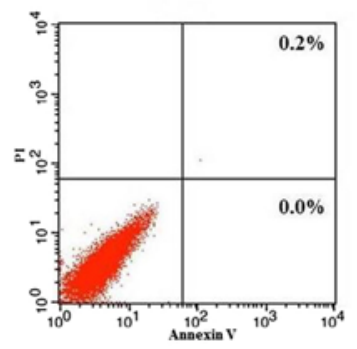

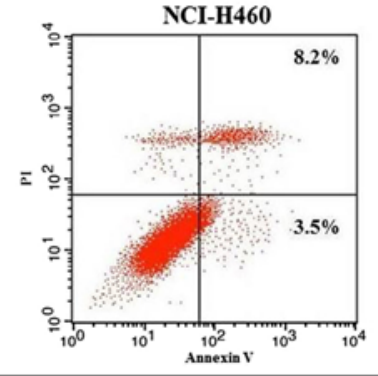

C
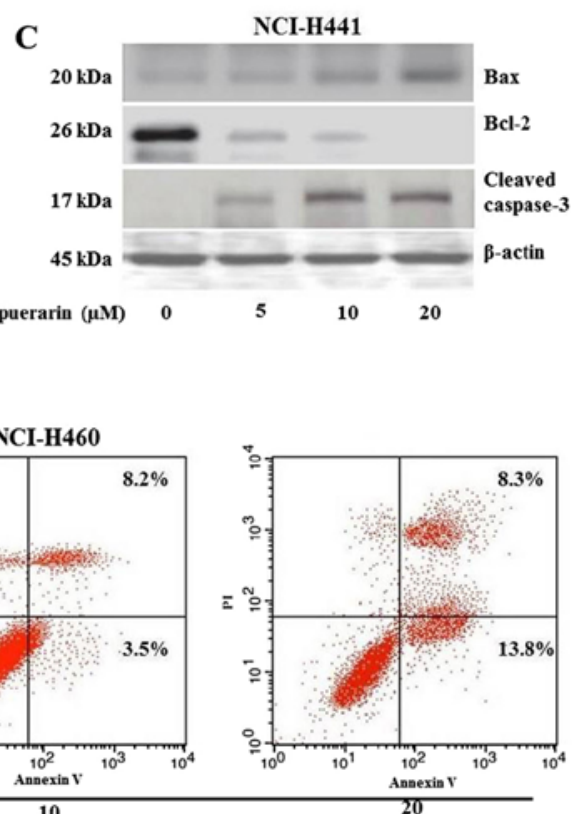

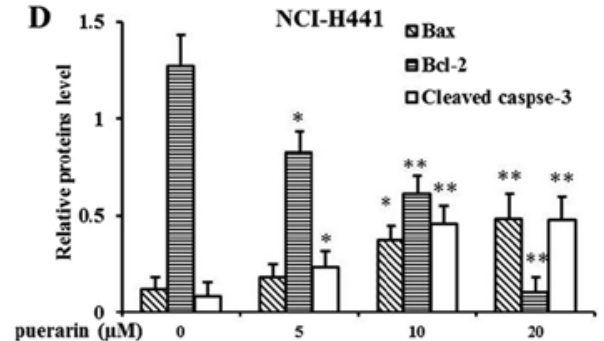

F

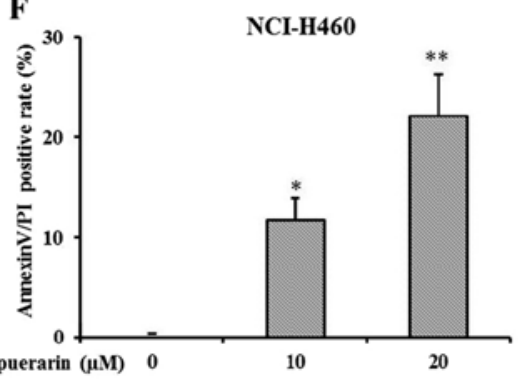

Figure 2. Puerarin promotes apoptosis in NCI-H441 or NCI-H460 cells. (A) NCI-H441 cells were treated with different concentrations of puerarin (5, 10 and $20 \mu \mathrm{M}$ ) for $72 \mathrm{~h}$. The apoptosis was determined by Annexin V/PI staining, and then was analyzed by flow cytometry (x-axis, Annexin V; y-axis, PI). (B) The quantification of the results of Annexin V/PI staining for NCI-H441 cells $\left({ }^{*} \mathrm{P}<0.05,{ }^{* *} \mathrm{P}<0.01\right.$, compared with DMSO control, $\mathrm{n}=3$ ). (C) Western blot analysis of Bax, Bcl-2 and cleaved caspase- 3 protein following exposure to puerarin. (D) Relative protein levels were quantified by using $\beta$-actin as control ( $\mathrm{P}<0.05$, ${ }^{* *} \mathrm{P}<0.01$, compared with DMSO control, $\left.\mathrm{n}=3\right)$. (E) NCI-H460 cells were treated with different concentrations of puerarin $(0,10$ and $20 \mu \mathrm{M})$ for $72 \mathrm{~h}$. The apoptosis was determined by Annexin V/PI staining, and then was analyzed by flow cytometry (x-axis, Annexin V; y-axis, PI). (F) The quantification of the results of Annexin V/PI staining for NCI-H460 cells $\left({ }^{*} \mathrm{P}<0.05,{ }^{* *} \mathrm{P}<0.01\right.$, compared with DMSO control, $\left.\mathrm{n}=3\right)$.

compared with the vehicle-treated cells (Fig. 1C). Moreover, cell morphology turned from a round shape into a spreading shape with good morphology, indicating that puerarin may not only inhibit cell growth, but also induce cell apoptosis. Similarly, puerarin also inhibited the growth of NCI-H460 cells in a time- and dose-dependent manner (Fig. 1D). Compared with the NCI-H460 cell line, NCI-H441 was more sensitive to puerarin. Therefore, the NCI-H441 cell line was selected for the following experiments.

Puerarin induces lung cancer cell apoptosis. Apoptosis is one of the predominant types of programmed cell death which involves a series of biochemical events leading to specific cell morphologic characteristics, including cell shrinkage, nuclear fragmentation, chromatin condensation and chromosomal DNA fragmentation. As observed in Fig. 1A and B, NCI-H441 cells appeared to exhibit apoptosis characteristics after puerarin treatment, thus, flow cytometric analysis by Annexin V and PI staining was performed to examine the proportion of apoptotic cells. Data indicated that puerarin treatment significantly increased the number of early apoptotic cells
(Annexin $\mathrm{V}^{+} / \mathrm{PI}^{-}$) and late apoptotic cells (Annexin $\mathrm{V}^{+} / \mathrm{PI}^{-}$) in a dose-dependent manner (Fig. 2A and B). In order to verify the apoptotic mechanism, the expression of the mitochondrial pathway proteins Bax and Bcl-2 were detected. As shown in Fig. 2C, the expression of Bax was upregulated along with increasing concentrations of puerarin. In contrast, the levels of Bcl-2 were decreased, leading to an increase in cleaved caspase-3. The relative protein levels normalized by $\beta$-actin are shown in Fig. 2D. Similarly, puerarin induced apoptosis in the NCI-H460 cells in a dose-dependent manner (Fig. 2E and F).

Puerarin slightly induces NCI-H44I cell autophagy. Since it has been reported that puerarin attenuates anoxia/reoxygenation injury (25) or prevents cardiac hypertrophy (26) through activation of autophagy, we investigated the relationship between puerarin and autophagy in tumor cells. Therefore, we detected the effect of puerarin treatment on NCI-H441 cell autophagy by using MDC staining assay. The positive staining of autophagosomes was measured via flow cytometry and the MDC-positive rate was calculated (Fig. 3A). There was a slight increase in the MDC-positive rate following 

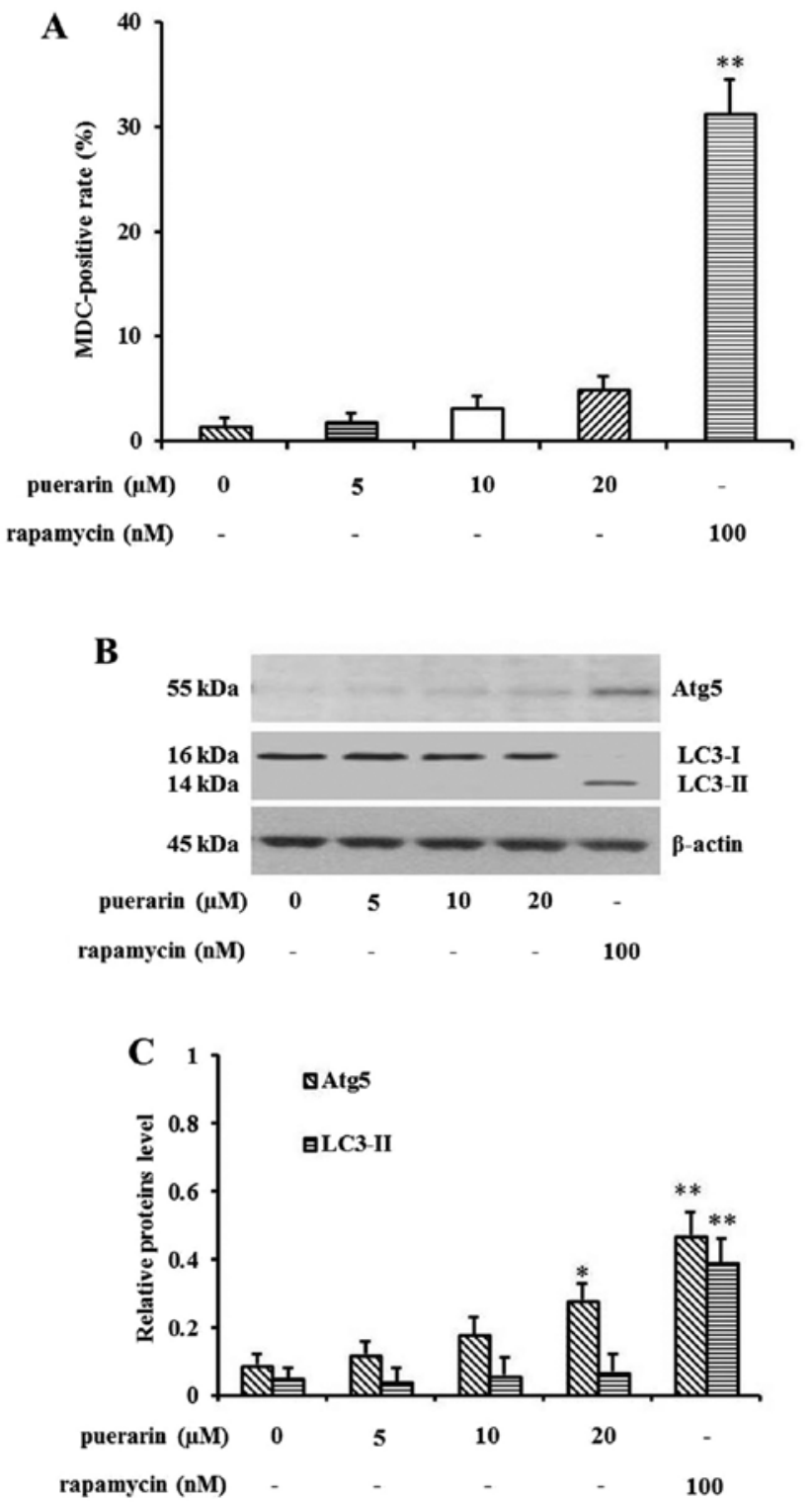

Figure 3. Puerarin induces lung cancer cell autophagy. (A) NCI-H441 cells were treated with different concentrations of puerarin $(5,10$ and $20 \mu \mathrm{M})$ for $72 \mathrm{~h}$. The autophagy was analyzed by flow cytometry, and the MDC-positive rate was calculated. Cells treated with $100 \mathrm{nM}$ of rapamycin was used as a positive control of autophagy $\left({ }^{* *} \mathrm{P}<0.01\right.$ compared with DMSO control, $\mathrm{n}=3$ ). (B) The expression of autophagy-related proteins Atg5 and LC3 were determined by western blot analysis and $\beta$-actin was used as loading control. (C) Relative protein levels were quantified by using $\beta$-actin as contro $\left(\right.$ $\mathrm{P}<0.05,{ }^{* *} \mathrm{P}<0.01$, compared with the DMSO control, $\mathrm{n}=3$ ).

puerarin treatment $(20 \mu \mathrm{M})$ compared with the control group. Rapamycin, a specific inhibitor of mTOR, was used as a positive control of autophagy. To further confirm puerarin-induced NCI-H441 cell autophagy, western blot analysis was performed to detect the alteration of essential molecules involved in autophagy. The expression of Atg5, autophagy protein 5, was gradually upregulated in a concentration-dependent manner. In contrast, the level of LC3-I, microtubule-associated protein 1 light chain 3 type I, was decreased. However, the expression of LC3-II, which correlates with the extent of autophagosomes, was not detectable in this cell line. Cells treated with rapamycin showed a strong signal of Atg5 and LC3-II, used as a positive control of autophagy (Fig. 3B and C).
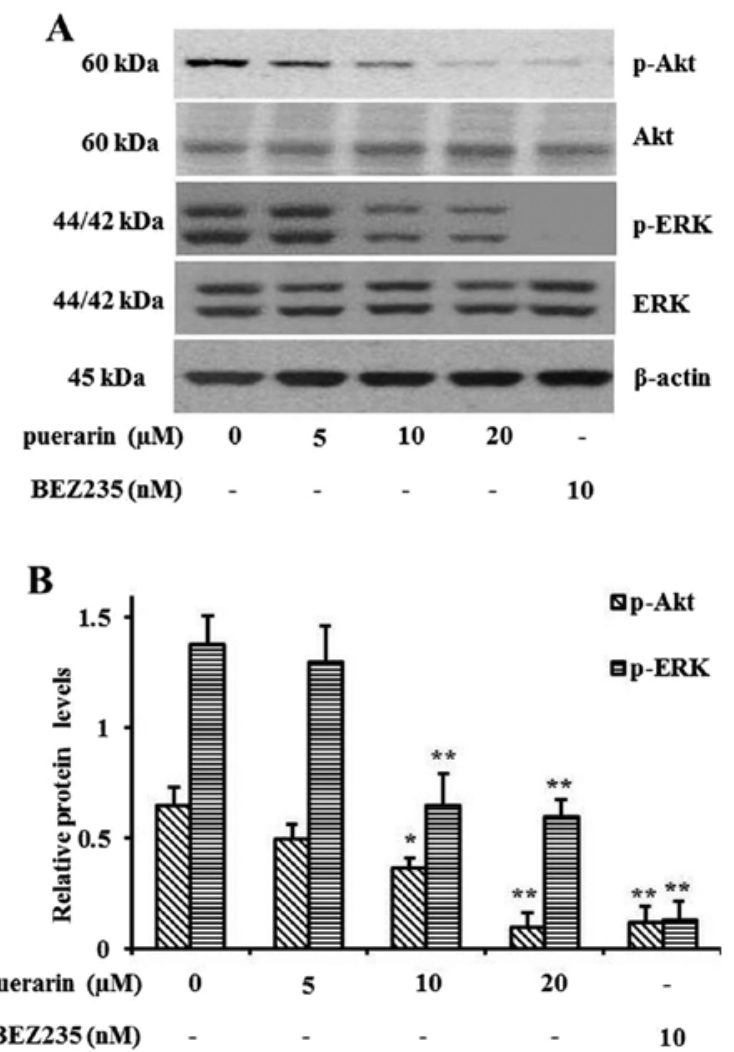

Figure 4. Effect of puerarin on the phosphorylation levels of Akt and ERK (A) Phosphorylation levels of Akt and ERK were assessed by western blot analysis in NCI-H441 cells after puerarin treatment for $72 \mathrm{~h}$. BEZ235 is the inhibitor of the PI3K pathway. (B) The relative protein levels were normalized by total Akt or ERK, respectively $\left({ }^{*} \mathrm{P}<0.05,{ }^{* *} \mathrm{P}<0.01\right.$ compared with the DMSO control, $n=3$ ).

Puerarin induces cell autophagy via the PI3K/Akt and ERK pathway. Since puerarin activated NCI-H441 cell autophagy, PI3K/Akt and ERK pathway activation, which are related to cell autophagy, was also determined by western blot analysis. As shown in Fig. 4A, the phosphorylation levels of Akt and ERK were extremely reduced after puerarin treatment. However, the total protein levels were not affected. BEZ235, an inhibitor of PI3K/mTOR, was used as a control for the inhibition of phosphorylation. The relative protein levels normalized by total Akt or ERK expression are shown in Fig. 4B.

Puerarin prevents tumor development in vivo. The effect of puerarin on the growth of primary tumor xenografts in nude mice was examined. Tumor volume was recorded every week. The volumes of the tumors in the treatment groups were significantly reduced in a dose-dependent manner compared with those in the control group, while the inhibition rate in the $100 \mathrm{mg} / \mathrm{kg}$ of puerarin treatment group was more highly significant than that in the other three groups (Fig. 5A). Representative figures of tumor dimensions in nude mice at the end of the experiment are shown in Fig. 5B. At the end of the experiment, the tumors were harvested and weighed in each group (Fig. 5C). In addition, there was no significant body weight loss in each group, which indicated that puerarin treatments were tolerable.

Detection of apoptotic cells in the xenograft tumor tissues. At the end of the experiment, 24 mice were necropsied, and 

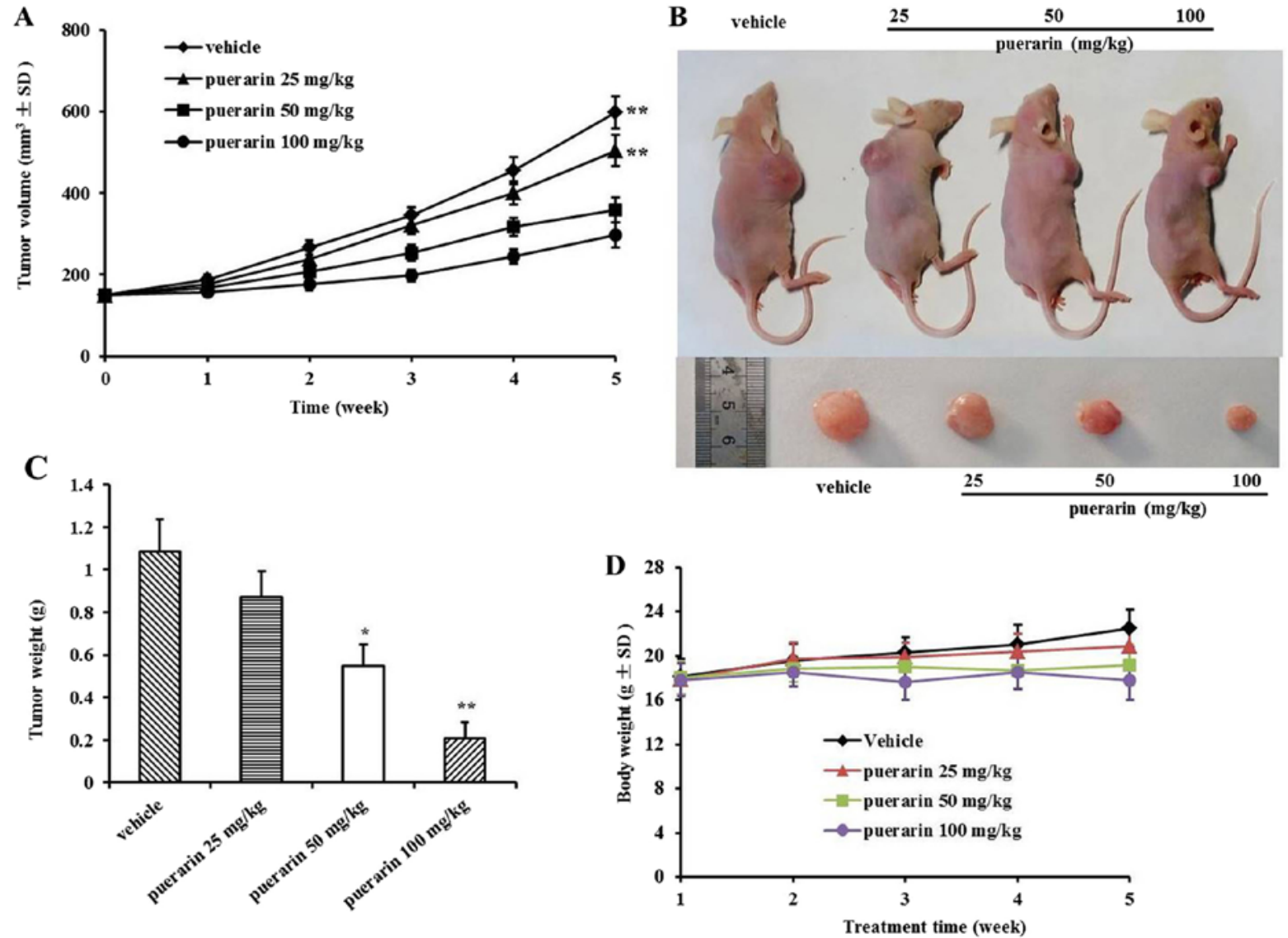

Figure 5. Effect of puerarin on tumor growth in xenograft nude mice. (A) Tumor volumes in xenograft tumors derived from NCI-H441 cells in nude mice were measured every week after treatment of puerarin $(25,50$ and $100 \mathrm{mg} / \mathrm{kg})$. Points indicate mean values $\left({ }^{* *} \mathrm{P}<0.01\right.$ compared with the vehicle control, $\left.\mathrm{n}=6\right)$. (B) Representative images of tumor dimensions at the end of the experiment. (C) Tumor weights at the end of the experiment $\left({ }^{*} \mathrm{P}<0.05,{ }^{* *} \mathrm{P}<0.01 \mathrm{compared}\right.$ with the vehicle control, $n=6$ ). (D) Body weights of mice were measured weekly ( $n=6)$.

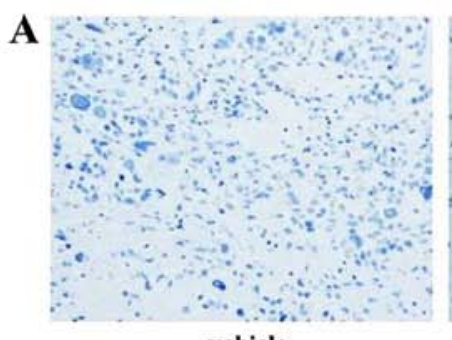

vehicle

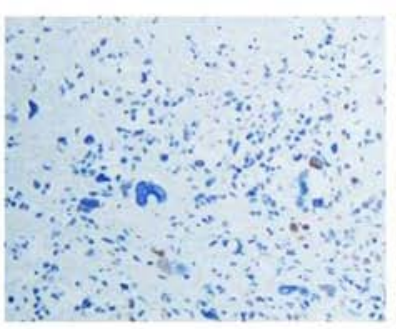

25

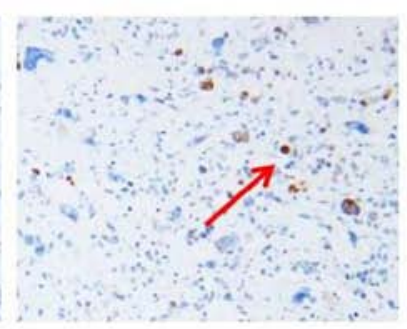

50

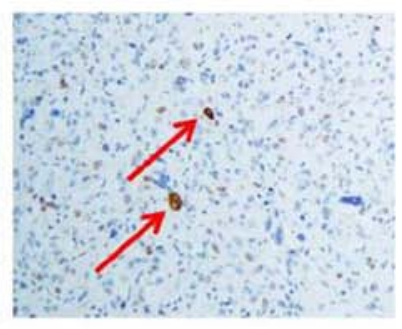

100

puerarin $(\mathrm{mg} / \mathrm{kg})$

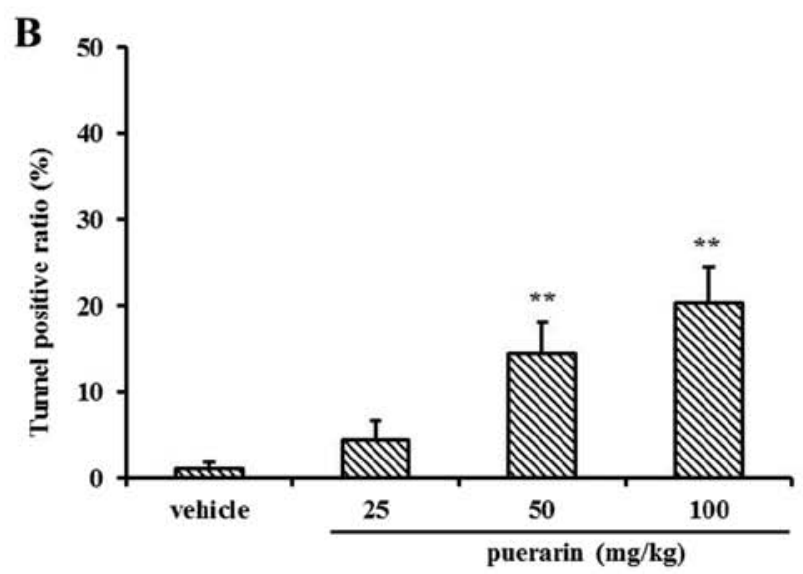

Figure 6. Puerarin induces cell apoptosis in xenograft nude mice. (A) Representative images of apoptotic cells detected in xenograft tumor tissues using TUNEL assay. Apoptotic cells appeared brown and are indicated by arrows (magnification, $\mathrm{x} 200$ ). (B) Quantified results of the TUNEL assay $\left({ }^{* *} \mathrm{P}<0.01, \mathrm{n}=3\right.$ ). 
the subcutaneous tumor tissue sections were used for detecting apoptotic cells by TUNEL assay. As shown in Fig. 6A, significant cell death had occurred in the tumor masses in the puerarin treatment groups, when compared with that of the vehicle group. The TUNEL-positive ratio was calculated by counting the positive-stained cells, which was then divided by the total cell number (Fig. 6B).

\section{Discussion}

Puerarin is the most important phytoestrogen extracted from the dried root of Pueraria lobata, which is a commonly used traditional Chinese herb. Researchers have concentrated on the pharmacological activities of puerarin, which displays a series of beneficial activities on hangover, cardiovascular disease, osteoporosis, neurological dysfunction, fever, and liver injury in clinical treatment and experimental research (27). In addition, more and more studies focus on the role of puerarin in cancer: puerarin inhibited cell growth and induced apoptosis in breast cancer cells (22), prevented human oophoroma cell invasion and metastasis (28), and induced neuroblastoma SH-SY5Y cell death by activating the PI3K/Akt pathway (29).

In the present study, we conducted an investigation of the effects of puerarin on NSCLC NCI-H441 cells. Puerarin inhibited cell growth in a dose and time-dependent manner, and promoted apoptosis as determined by Annexin V/PI staining. In addition, puerarin treatment increased the active form of caspase-3 (cleaved caspase-3), with an increase in Bax expression and a decrease in Bcl-2 levels. The Bcl-2 family of proteins plays an important role in the regulation of apoptosis in many cellular systems, by either inhibiting (Bcl-2, Bcl-XL, Bcl-W, Bfl-1, and Mcl-1) or promoting apoptosis (Bax, Bak, Bad, Bcl-Xs, Bid, and Hrk) (30). Heterodimerization between pro- and anti-apoptotic members of this family and relative levels of both types of proteins may determine the susceptibility to a given apoptotic stimulus and cell fate $(31,32)$.

Moreover, a high concentration of puerarin induced NCI-H441 cell autophagy as assessed by MDC staining, and caused a slight increase in Atg5 expression and reduction in the LC3-I protein level. Notably, there was no detectable alteration of LC3-II expression compared with the control. During autophagy, the nascent proMAP1LC3/LC3 (microtubule-associated protein 1 light chain 3 ) is processed post-translationally into the cytoplasmatic soluble form LC3-I and by conjugation to phosphatidylethanolamine (PE), generating the membraneassociated LC3-II puncta, which correlates with the extent of autophagosomes (33). For this reason, LC3 is a commonlyused autophagic marker (34). We failed to detect a change in LC3-II expression, perhaps because the samples used for this experiment were in the early stage of autophagy. To confirm our hypothesis, we analyzed the phosphorylation levels of Akt and ERK, since it is known that PI3K/Akt and ERK pathway inactivation is required during cell autophagy. Indeed, the phosphorylation levels of Akt and ERK were reduced in a dose-dependent manner, further indicating that puerarin treatment induced NCI-H441 cell autophagy. In addition, puerarin prevented tumor growth by inducing tumor cell apoptosis in the xenograft nude mouse model. This result was consistent with in vitro data.
Taken together, puerarin, inhibited cell growth, induced apoptosis and autophagy and could be considered as a possible chemotherapeutic drug for the treatment of lung cancer.

\section{Acknowledgements}

This study was supported by the Key Laboratory for Chemistry and Molecular Engineering of Medicinal Resources (Guangxi Normal University) (CMEMR2016-B01).

\section{References}

1. Ferlay J, Soerjomataram I, Dikshit R, Eser S, Mathers C, Rebelo M, Parkin DM, Forman D and Bray F: Cancer incidence and mortality worldwide: Sources, methods and major patterns in GLOBOCAN 2012. Int J Cancer 136: E359-E386, 2015.

2. Świątkowska B,SzubertZ, Sobala W and Szeszenia-Dąbrowska N: Predictors of lung cancer among former asbestos-exposed workers. Lung Cancer 89: 243-248, 2015.

3. Devesa SS, Bray F, Vizcaino AP and Parkin DM: International lung cancer trends by histologic type: male:female differences diminishing and adenocarcinoma rates rising. Int J Cancer 117: 294-299, 2005.

4. Howlader N, Noone AM, Krapcho M, Garshell J, Miller D, Altekruse SF, Kosary CL, Yu M, Ruhl J, Tatalovich Z, Mariotto A, Lewis DR, Chen HS, Feuer EJ, Cronin KA (eds). SEER Cancer Statistics Review, 1975-2012, National Cancer Institute. Bethesda, MD, http://seer.cancer.gov/csr/1975_2012/, based on November 2014 SEER data submission, posted to the SEER web site, April 2015.

5. Allemani C, Weir HK, Carreira H, Harewood R, Spika D, Wang XS, Bannon F, Ahn JV, Johnson CJ, Bonaventure A, et al; CONCORD Working Group: Global surveillance of cancer survival 1995-2009: Analysis of individual data for 25,676,887 patients from 279 population-based registries in 67 countries (CONCORD-2). Lancet 385: 977-1010, 2015.

6. Gupta SK: Role of Crizotinib in previously treated non-smallcell lung cancer. South Asian J Cancer 3: 138-140, 2014.

7. Schiller JH, Harrington D, Belani CP, Langer C, Sandler A, Krook J, Zhu J and Johnson DH; Eastern Cooperative Oncology Group: Comparison of four chemotherapy regimens for advanced non-small-cell lung cancer. N Engl J Med 346: 92-98, 2002.

8. Angulo P, Kaushik G, Subramaniam D, Dandawate P, Neville K, Chastain $\mathrm{K}$ and Anant $\mathrm{S}$ : Natural compounds targeting major cell signaling pathways: A novel paradigm for osteosarcoma therapy. J Hematol Oncol 10: 10, 2017.

9. ChanvorachoteP,ChamniS,NinsontiaCandPhiboonchaiyananPP: Potential anti-metastasis natural compounds for lung lancer. Anticancer Res 36: 5707-5717, 2016.

10. Shanmugam MK, Lee JH, Chai EZ, Kanchi MM, Kar S, Arfuso F, Dharmarajan A, Kumar AP, Ramar PS, Looi CY, et al: Cancer prevention and therapy through the modulation of transcription factors by bioactive natural compounds. Semin Cancer Biol 40-41: 35-47, 2016.

11. Pan MH, Lai CS, Wu JC and Ho CT: Molecular mechanisms for chemoprevention of colorectal cancer by natural dietary compounds. Mol Nutr Food Res 55: 32-45, 2011.

12. Guo XF, Yang ZR, Wang J, Lei XF, Lv XG and Dong WG: Synergistic antitumor effect of puerarin combined with 5-fluorouracil on gastric carcinoma. Mol Med Rep 11: 2562-2568, 2015.

13. Zeng YP, Yang ZR, Guo XF, Jun W and Dong WG: Synergistic effect of puerarin and 5-fluorouracil on hepatocellular carcinoma. Oncol Lett 8: 2436-2442, 2014.

14. Zhang SF, Wang XL, Yang XQ and Chen N: Autophagyassociated targeting pathways of natural products during cancer treatment. Asian Pac J Cancer Prev 15: 10557-10563, 2014.

15. Yanagihara K, Ito A, Toge T and Numoto M: Antiproliferative effects of isoflavones on human cancer cell lines established from the gastrointestinal tract. Cancer Res 53: 5815-5821, 1993.

16. Tian F, Xu LH, Zhao W, Tian LJ and Ji XL: The optimal therapeutic timing and mechanism of puerarin treatment of spinal cord ischemia-reperfusion injury in rats. J Ethnopharmacol 134: 892-896, 2011.

17. Wong KH, Li GQ, Li KM, Razmovski-Naumovski V and Chan K: Kudzu root: Traditional uses and potential medicinal benefits in diabetes and cardiovascular diseases. J Ethnopharmacol 134: 584-607, 2011. 
18. Yuan Y, Zong J, Zhou H, Bian ZY, Deng W, Dai J, Gan HW, Yang Z, Li H and Tang QZ: Puerarin attenuates pressure overload-induced cardiac hypertrophy. J Cardiol 63: 73-81, 2014

19. Yeung DK, Leung SW, Xu YC, Vanhoutte PM and Man RY: Puerarin, an isoflavonoid derived from Radix puerariae, potentiates endothelium-independent relaxation via the cyclic AMP pathway in porcine coronary artery. Eur J Pharmacol 552: 105-111, 2006.

20. $\mathrm{Yu} \mathrm{Z}$ and $\mathrm{Li} \mathrm{W}$ : Induction of apoptosis by puerarin in colon cancer HT-29 cells. Cancer Lett 238: 53-60, 2006.

21. Wang Y, Ma Y, Zheng Y, Song J, Yang X, Bi C, Zhang D and Zhang Q: In vitro and in vivo anticancer activity of a novel puerarin nanosuspension against colon cancer, with high efficacy and low toxicity. Int J Pharm 441: 728-735, 2013.

22. Zhang WG, Liu XF, Meng KW and Hu SY: Puerarin inhibits growth and induces apoptosis in SMMC-7721 hepatocellular carcinoma cells. Mol Med Rep 10: 2752-2758, 2014.

23. Lin YJ, Hou YC, Lin CH, Hsu YA, Sheu JJ, Lai CH, Chen BH, Lee Chao PD, Wan L and Tsai FJ: Puerariae radix isoflavones and their metabolites inhibit growth and induce apoptosis in breast cancer cells. Biochem Biophys Res Commun 378: 683-688, 2009

24. National Research Council of the National Academies: Guide for the Care and Use of Laboratory Animals: 2011. The National Academies Press, Washington, DC, 2011.

25. Ma Y, Gai Y, Yan J, Li J and Zhang Y: Puerarin attenuates anoxia/ reoxygenation injury through enhancing $\mathrm{Bcl}-2$ associated athanogene 3 Expression, a modulator of apoptosis and autophagy. Med Sci Monit 22: 977-983, 2016

26. Liu B, Wu Z, Li Y, Ou C, Huang Z, Zhang J, Liu P, Luo C and Chen M: Puerarin prevents cardiac hypertrophy induced by pressure overload through activation of autophagy. Biochem Biophys Res Commun 464: 908-915, 2015.
27. Wei SY, Chen Y and Xu XY: Progress on the pharmacological research of puerarin: A review. Chin J Nat Med 12: 407-414, 2014.

28. Han J, Yu CQ and Shen W: Inhibitory effects of puerarin on invasion and metastasis of oophoroma cells HO-8910. Zhongguo Zhong Xi Yi Jie He Za Zhi 29: 632-635, 2009 (In Chinese).

29. Zhu G, Wang X, Wu S and Li Q: Involvement of activation of PI3K/Akt pathway in the protective effects of puerarin against $\mathrm{MPP}^{+}$-induced human neuroblastoma SH-SY5Y cell death. Neurochem Int 60: 400-408, 2012.

30. Gross A, McDonnell JM and Korsmeyer SJ: BCL-2 family members and the mitochondria in apoptosis. Genes Dev 13 1899-1911, 1999.

31. Oltvai ZN, Milliman CL and Korsmeyer SJ: Bcl-2 heterodimerizes in vivo with a conserved homolog, Bax, that accelerates programmed cell death. Cell 74: 609-619, 1993.

32. Choi YH, Kong KR, Kim YA, Jung KO, Kil JH, Rhee SH and Park KY: Induction of Bax and activation of caspases during beta-sitosterol-mediated apoptosis in human colon cancer cells Int J Oncol 23: 1657-1662, 2003.

33. Kabeya Y, Mizushima N, Ueno T, Yamamoto A, Kirisako T, Noda T, Kominami E, Ohsumi Y and Yoshimori T: LC3, a mammalian homologue of yeast Apg8p, is localized in autophagosome membranes after processing. EMBO J 19: 5720-5728, 2000.

34. Klionsky DJ, Abdelmohsen K, Abe A, Abedin MJ, Abeliovich H, Acevedo Arozena A, Adachi H, Adams CM, Adams PD, Adeli K, et al: Guidelines for the use and interpretation of assays for monitoring autophagy (3rd edition). Autophagy 12: 1-222, 2016. 